\title{
FENOLOGIA REPRODUTIVA, SÍNDROMES DE POLINIZAÇÃO E DISPERS̃̃O EM ESPÉCIES de LEguminosae dos CAMPOS RUPESTRES do PARQUe Estadual do Itacolomi, Minas Gerais, BrasiL ${ }^{1}$
}

\author{
Valquíria Ferreira Dutra ${ }^{2,4}$, Milene Faria Vieira ${ }^{2}$, \\ Flávia Cristina Pinto Garcia ${ }^{2} \&$ Haroldo Cavalcante de Lima ${ }^{3}$
}

\begin{abstract}
Resumo
(Fenologia reprodutiva, síndromes de polinização e dispersão em espécies de Leguminosae dos campos rupestres do Parque Estadual do Itacolomi, Minas Gerais, Brasil) A fenologia reprodutiva e as síndromes florais e de dispersão em 45 táxons de Leguminosae foram estudadas nos campos rupestres do Parque Estadual do Itacolomi (PEI), Minas Gerais, Brasil, entre setembro/2003 e outubro/2004. A floração na comunidade ocorreu durante todo o período de estudo, com o maior pico observado em março (66,6\% dos táxons), havendo correlação positiva com a precipitação e a temperatura. A maioria dos táxons estudados $(88,8 \%)$ apresentou características florais da síndrome de melitofilia. Foram descritos, para as espécies de Chamaecrista, dois padrões de deposição de pólen no corpo do polinizador, o direto e o indireto. Houve frutificação durante todo o período de estudo, com o maior pico em abril (57,7\%), havendo correlação significativa dessa fenofase com a temperatura. A autocoria foi a síndrome de dispersão mais frequente $(66,6 \%)$, associada principalmente aos frutos dos tipos legume e folículo. Os resultados indicam que a sazonalidade climática é importante para a floração, frutificação e dispersão dos diásporos nos campos rupestres do PEI e reforçam a importância das espécies de Leguminosae como uma importante fonte de recursos alimentares (pólen e néctar) para as abelhas.
\end{abstract}

Palavras-chave: autocoria, melitofilia, campo rupestre, Chamaecrista.

\section{Abstract}

(Reproductive phenology, pollination and dispersal syndromes in Leguminosae from the campos rupestres of Itacolomi State Park, Minas Gerais, Brazil) The reproductive phenology, floral and dispersal syndrome were studied in 45 taxa of Leguminosae from the "campos rupestres" of the Itacolomi State Park, Minas Gerais, Brazil, from September/2003 to October/2004. Flowering, in the community, occurred throughout the study period, with the highest peak in March (66.6\% of taxons), displaying positive correlation with rainfall and temperature. Most of the studied taxa (88.8\%) presented floral characteristics of bee-pollination syndrome. Two patterns of pollen deposition on the pollinator's body were described for Chamaecrista species; direct and indirect Taxa were observed fruiting throughout the study period, with the highest peak in April (57.7\%), with this phenophase displaying significant correlation with temperature. Autochory was the most frequent dispersal syndrome (66.6\%), mainly represented by legume- and follicle-type fruits. The results indicate that the climatic seasonality is important for the flowering, fruiting and dispersal of the diaspores in the "campos rupestres" of the Itacolomi State Park and emphasize the importance of the Leguminosae as an important source of food resources (pollen and nectar) for the bees.

Key words: autochory, mellitophilous syndrome, campos rupestres, Chamaecrista.

\section{INTRODUÇÃo}

As interações entre plantas e seus polinizadores, dispersores e o ambiente são importantes para o entendimento da estrutura e da dinâmica de comunidades vegetais (Frankie et al. 1974; Fournier 1976; Yamamoto et al. 2007), essenciais para subsidiar estratégias de conservação da biodiversidade (Wunderlee 1997). Dentro deste contexto, os eventos fenológicos, tais como floração e frutificação (Rathcke \& Lacey 1985), exercem influência sobre os recursos disponíveis para

Artigo recebido em 06/2008. Aceito para publicação em 04/2009.

${ }^{1}$ Parte da Dissertação de Mestrado da primeira autora. Curso de Pós-Graduação em Botânica, Universidade Federal de Viçosa, MG, Brasil

${ }^{2}$ Universidade Federal de Viçosa, Depto. Biologia Vegetal, 36570-000, Viçosa, MG, Brasil

${ }^{3}$ Instituto de Pesquisas Jardim Botânico do Rio de Janeiro, R. Pacheco Leão 915, 22460-030, Rio de Janeiro, RJ, Brasil

${ }^{4}$ Autor para correspondência: valquiria.dutra@bol.com.br 
muitos organismos (Conceição et al. 2007); e a diversidade das síndromes florais mostra a riqueza de interações entre flores e polinizadores, sendo importante na compreensão dos mecanismos de diversificação das características florais (Yamamoto et al. 2007).

Em comunidades vegetais tropicais, especialmente nas campestres, o estrato herbáceo-subarbustivo ainda é pouco estudado em relação à fenologia e às síndromes florais e de dispersão (p.ex. Mantovani \& Martins 1988; Batalha et al. 1997; Batalha \& Mantovani 2000; Carmo \& Franceschinelli 2002; Munhoz \& Felfili 2005; Conceição et al. 2007). Nos campos rupestres esses estudos estão restritos aos da Chapada Diamantina, na Bahia (Conceição et al. 2007) e aos de Brumadinho (Carmo \& Franceschinelli 2002), Serra do Cipó (Madeira \& Fernandes 1999) e campos ferruginosos do Parque Estadual do Itacolomi (Dutra et al. 2005), em Minas Gerais. Observa-se que, apesar dos campos rupestres ocuparem áreas consideradas de alta a extrema importância biológica, devido à riqueza da flora e ao alto índice de endemismos (Harley 1995; Drummond et al. 2005), pouco se conhece sobre as interações entre suas plantas e a fauna antófila, e sobre os mecanismos de dispersão de diásporos. Vale ressaltar que essa formação vegetacional apresenta-se adaptada a situações de estresse, como ventos fortes, elevada insolação, grande amplitude térmica diurna/noturna e déficit hídrico (Menezes \& Giulietti 1986; Giulietti et al. 1987), que devem refletir nestas interações e mecanismos.

Leguminosae está subdividida em três subfamílias, Caesalpinioideae, Mimosoideae e Papilionoideae, cada uma com características florais distintas e bem elaboradas, que resultaram numa diversidade de mecanismos de polinização (Stirton 1981). É uma das famílias mais representativas e diversas dos campos rupestres, representada por cerca de 340 espécies (Romero 2002; Dutra et al. 2008). Apesar dessa riqueza, apenas os trabalhos com espécies de Chamaecrista (Madeira \& Fernandes 1999) e de táxons de Papilionoideae (Dutra et al. 2005), tratam da fenologia de
Leguminosae nestes campos. Trabalhos sobre as síndromes florais são inexistentes.

O objetivo deste estudo foi fornecer informações sobre a fenologia reprodutiva e as síndromes florais e de dispersão das espécies de Leguminosae que ocorrem nos campos rupestres do Parque Estadual do Itacolomi, estado de Minas Gerais.

\section{Material e Métodos}

O estudo foi realizado entre setembro de 2003 e outubro de 2004, no Parque Estadual do Itacolomi (PEI), localizado nos municípios de Ouro Preto e Mariana, Minas Gerais

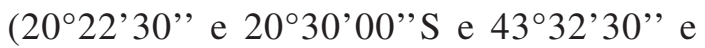
$\left.43^{\circ} 22^{\prime} 30^{\prime \prime} \mathrm{W}\right)$. A vegetação do PEI ocupa a zona de transição entre os domínios reais da floresta atlântica e do cerrado, sendo caracterizada como floresta estacional semidecidual e campo rupestre (Messias et al. 1997), este último abrangendo a área localizada acima da cota de $1.200 \mathrm{~m}$ de altitude (Peron 1989). O clima é sazonal, do tipo Cwb, segundo Köppen (Messias et al. 1997), com duas estações bem definidas: uma seca e fria, de maio a outubro, e outra quente e úmida, de novembro a abril (Fig. 1a). No período de estudo, a temperatura média foi de $19^{\circ} \mathrm{C}$ e a precipitação média mensal $125 \mathrm{~mm}$.

Foram estudados 45 táxons específicos e infra-específicos de Leguminosae, segundo o levantamento florístico realizado por Dutra (2005), sendo nove táxons de Mimosoideae, 10 de Caesalpinioideae e 26 de Papilionoideae (Tab. 1).

Foram registradas mensalmente a presença/ausência de floração (presença de botões florais ou de flores abertas) e de frutificação (presença de frutos jovens ou maduros) nos táxons estudados. A análise qualitativa das estratégias de floração e frutificação foi realizada utilizando-se os padrões de Newstrom et al. (1994), em: contínua (ao longo do ano, com breve ou nenhuma interrupção), anual breve (menor do que um mês), anual intermediária (de um a cinco meses), anual estendida (de mais de cinco 


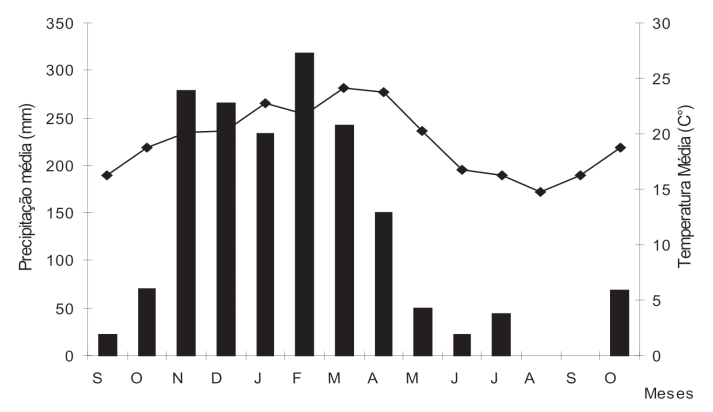

a
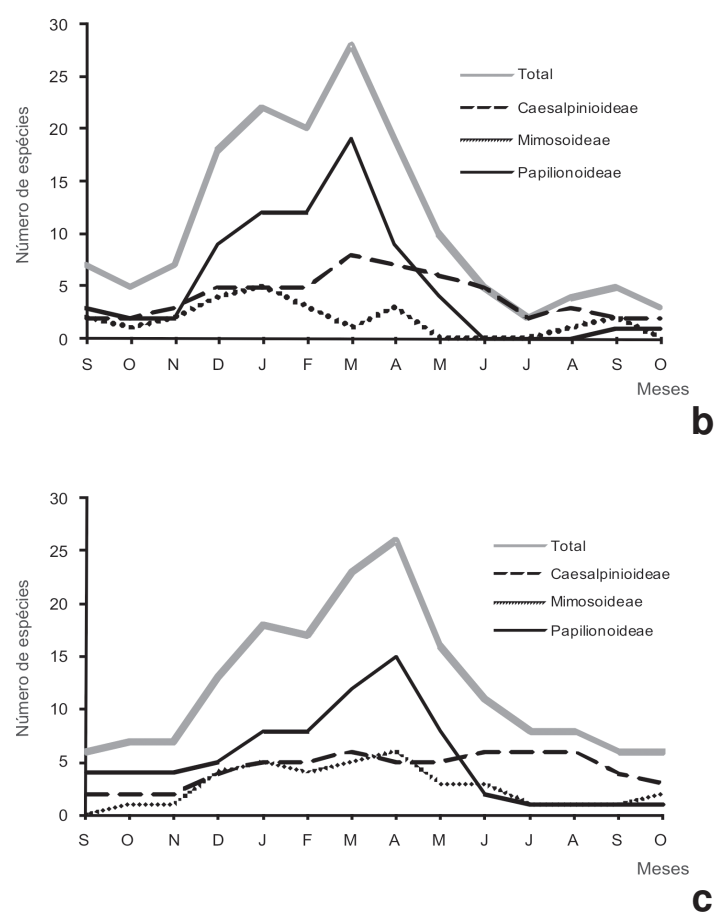

Figura 1 - a. Médias mensais de precipitação (•) e temperatura (- -), no período de estudo, no Município de Ouro Preto, Minas Gerais; b. número de espécies de Leguminosae em floração; c. número de espécies de Leguminosae em frutificação. (Fonte: Estação Meteorológica da ALCAN, Saramenha).

Figure $1-a$. Mean values of rainfall precipitation (-) and temperature (- - ), per month, along the study period, at the municipality of Ouro Preto, Minas Gerais; b. number of Leguminosae species with flower; c. number of Leguminosae species with fruits. (Source: Meteorological station of ALCAN, Saramenha).

meses), supra-anual (menos de um ciclo por ano) e subanual (mais de um ciclo por ano). Foi testada a relação entre as fenofases e as médias mensais de precipitação e de temperatura, através do coeficiente de Pearson (Morettin \& Bussab 2004).
Foram registrados a simetria e a forma da corola, a coloração da corola ou dos filetes, o horário de abertura das flores e o recurso floral coletado pelos visitantes e, a partir destas características, inferidas as síndromes florais dos táxons estudados (sensu Faegri \& van der Pijl 1979; Gottsberger \& Silberbauer-Gottsberger 1988; Endress 1994).

Durante o período de floração, visitantes, como abelhas, foram capturados e beija-flores apenas registrados. Os insetos foram coletados com auxílio de puçá, mortos e imediatamente analisados para verificar o local de deposição de pólen em seus corpos. Posteriormente, foram montados em alfinetes entomológicos, etiquetados, identificados e depositados no Museu Regional de Entomologia da Universidade Federal de Viçosa.

A caracterização das síndromes de dispersão foi baseada na morfologia dos diásporos e foram identificadas segundo a classificação proposta por van der Pijl (1982).

Nos táxons que não floriram ou não frutificaram, as características florais e as síndromes de dispersão foram inferidas de acordo com a literatura.

Nas espécies do gênero Chamaecrista, foi observada a existência de grupos distintos de espécies, considerando as diferenças das alturas dos estames e do pistilo. Estas diferenças foram utilizadas para descrever padrões de deposição de pólen no corpo dos polinizadores, ou seja, deposição direta e deposição indireta, que auxiliaram no entendimento dos mecanismos de polinização dessas plantas. Para isso, foram realizadas medidas das alturas dos estames e do pistilo e obtidas as diferenças proporcionais $(\mathrm{dp})$ entre elas.

\section{Resultados}

A maioria dos táxons apresenta hábito arbustivo $(57,7 \%)$, seguido pelo herbáceo $(24,4 \%)$ e trepador $(17,9 \%$; sendo $15,5 \%$ de trepadeiras e $2,4 \%$ de lianas) (Tab. 1). 
Tabela 1 - Leguminosae estudadas nos campos rupestres do Parque Estadual do Itacolomi, Minas Gerais, Brasil - hábito, características florais e padrões fenológicos. $\mathrm{hb}=$ hábito $(\mathrm{ab}=$ arbusto, ev = erva, $\mathrm{ln}=$ liana, $\mathrm{t} \mathrm{p}=$ trepadeira $)$; $\mathrm{cr}=$ cor das pétalas ou filetes $(\mathrm{am}=\mathrm{amarelo}$, av = azulvioláceo, $\mathrm{br}=$ branco, $\mathrm{cm}=$ creme-esverdeada, $\mathrm{ll}=$ lilás, $\mathrm{rs}=$ róseo, $\mathrm{vm}=$ vermelho $) ; \mathrm{rf}=$ recurso floral $(\mathrm{nc}=$ néctar, $\mathrm{pl}=\mathrm{pólen}, \mathrm{pn}=\mathrm{pólen} \mathrm{e}$ néctar); $\mathrm{sf}=$ síndrome floral (es = esfingofilia, $\mathrm{ml}=$ melitofilia, on = ornitofilia, $\mathrm{ps}=$ psicofilia, $\mathrm{qr}=$ quiropterofilia); $\mathrm{tf}=$ tipo de fruto $(\mathrm{cp}=\mathrm{craspédio}$, $\mathrm{dp}=$ drupa, $\mathrm{fl}=$ folículo, $\mathrm{lc}=$ legume nucóide, $\mathrm{lg}=$ legume, $\mathrm{lm}=$ lomento, $\mathrm{sm}=$ sâmara $) ; \mathrm{dd}=$ síndrome de dispersão dos diásporos $($ an $=$ anemocórica, at = autocórica, zo = zoocórica), •floração, ๑frutificação, ఐestação seca, «estação chuvosa.

Table 1 - Leguminosae from the "campos rupestres" of Parque Estadual do Itacolomi, Minas Gerais, Brasil. Habit, flower traits and phenological patterns. hb $=$ habit $(\mathrm{ab}=$ shrub, ev = herb, $\ln =$ liana, $\mathrm{tp}=$ climbing vine $), \mathrm{cr}=$ petal or filament color $(\mathrm{am}=$ yellow, av = violet-blue, $\mathrm{br}=\mathrm{white}, \mathrm{cm}=\mathrm{greenish}-\mathrm{cream}, \mathrm{ll}=$ lilac $\mathrm{rs}=$ pinkish, $\mathrm{vm}=\mathrm{red}), \mathrm{rf}=$ rewards $(\mathrm{nc}=$ nectar, $\mathrm{pl}=$ pollen, $\mathrm{pn}=$ pollen and nectar $), \mathrm{sf}=$ pollination syndrome $(\mathrm{es}=$ sphingophily, $\mathrm{ml}=$ mellittophily, on $=$ ornitophily, $\mathrm{ps}=$ psicophily, $\mathrm{qr}=$ chiropterophily $), \mathrm{tf}=$ fruit type $(\mathrm{cp}=$ craspedium, $\mathrm{dp}=$ drupe, $\mathrm{fl}=$ follicle, $\mathrm{lc}=$ "nucóide" legume, $\mathrm{lg}=\mathrm{legume}, \mathrm{lm}=\mathrm{loment}, \mathrm{sm}$ = samara $), \mathrm{dd}=$ dispersion syndromes $(\mathrm{an}=$ anemocory, at = autocory, $\mathrm{zo}=$ zoocory), •flowering, $\odot$ fruiting, $\square$ dry season, $\square$ rainy season.

\begin{tabular}{|c|c|c|}
\hline \multirow{2}{*}{ Táxons específicos e infra-específicos (número de tombo VIC) } & \multirow{2}{*}{ hb cr rf $\mathrm{sf}$ tf $\mathrm{dd}$} & Fenologia reprodutiva \\
\hline & & S O N D J F M A M J J A S O \\
\hline Caesalpinioideae & & \\
\hline Chamaecrista dentata (Vogel) H.S. Irwin \& Barneby (28292) & $\mathrm{ab}$ am $\mathrm{pl} \mathrm{ml} \lg$ at & 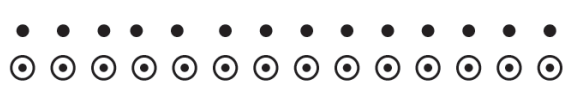 \\
\hline Chamaecrista mucronata (Spreng.) H.S. Irwin \& Barneby (28295) & $\mathrm{ab}$ am $\mathrm{pl} \mathrm{ml} \lg$ at & 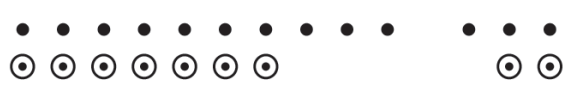 \\
\hline Chamaecrista trichopoda (Benth.) Britton \& Rose ex Britton \& Killip (28986) & $\mathrm{ab}$ am $\mathrm{pl} \mathrm{ml} \lg$ at & 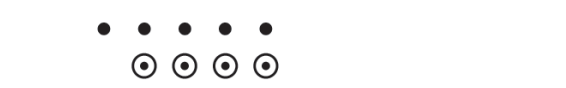 \\
\hline Senna reniformis (G. Don) H.S. Irwin \& Barneby (28296) & $\mathrm{ab}$ am $\mathrm{pl} \mathrm{ml} \lg$ at & 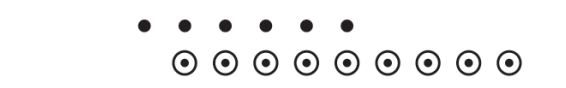 \\
\hline Chamaecrista desvauxii var. langsdorfii (Kunth ex Vogel) H.S. Irwin \& Barneb & ab am pl ml $\lg$ at & 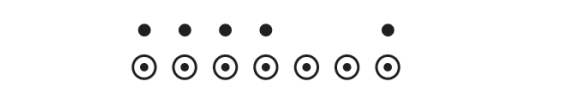 \\
\hline Chamaecrista rotundifolia var. rotundifolia (Pers.) Greene (28989) & ev am $\mathrm{pl} \mathrm{ml} \lg$ at & $\bullet$ \\
\hline Chamaecrista hedysaroides (Vogel) H.S. Irwin \& Barneby (28959) & $\mathrm{ab}$ am $\mathrm{pl} \mathrm{ml} \mathrm{lg}$ at & $\bullet \cdot \odot \odot \odot \odot \odot$ \\
\hline Senna pendula var. glabrata (Vogel) H.S. Irwin \& Barneby (28317) & $\mathrm{ab}$ am $\mathrm{pl} \mathrm{ml} \mathrm{lg}$ at & $\bullet \bullet \odot \odot \odot$ \\
\hline
\end{tabular}




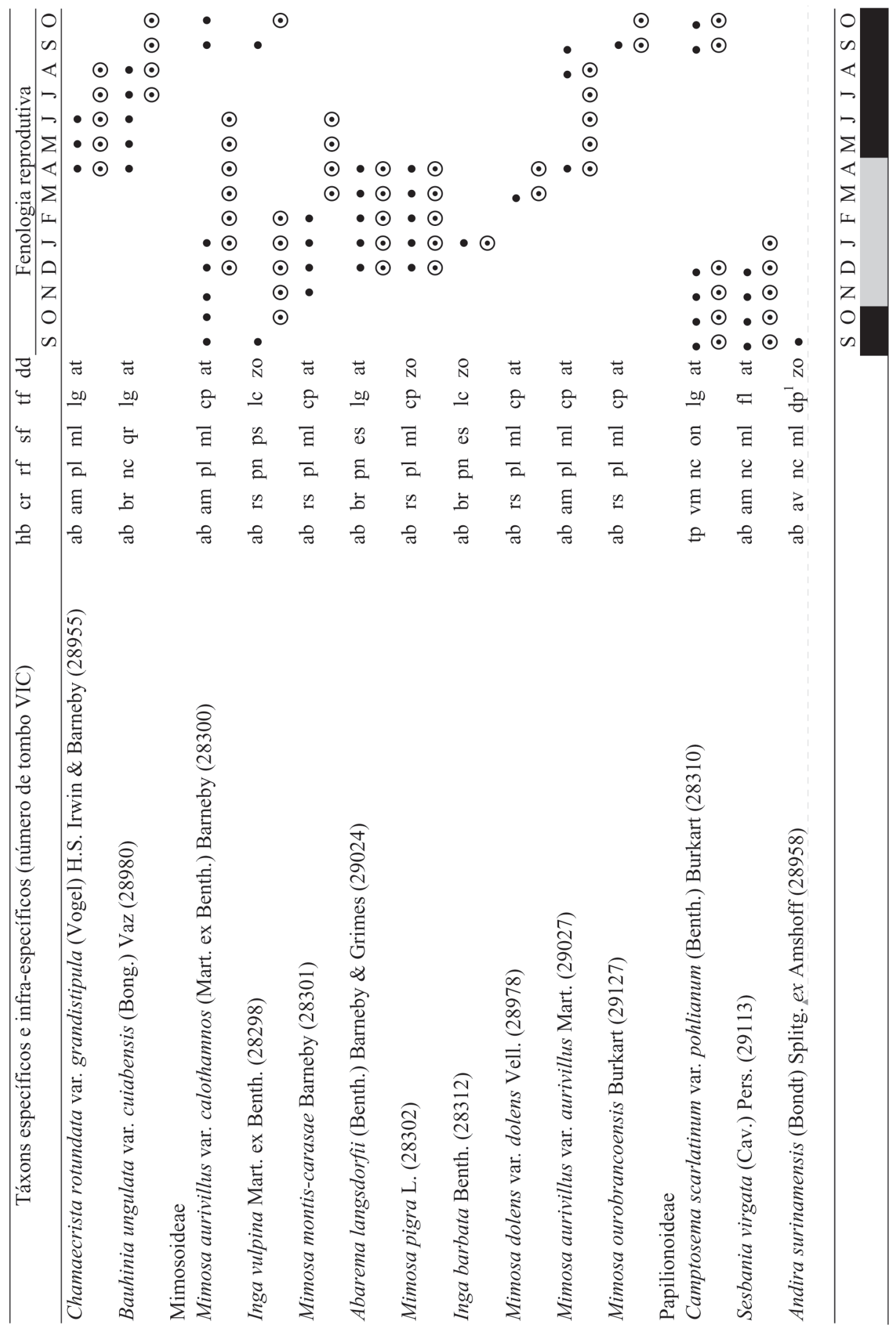




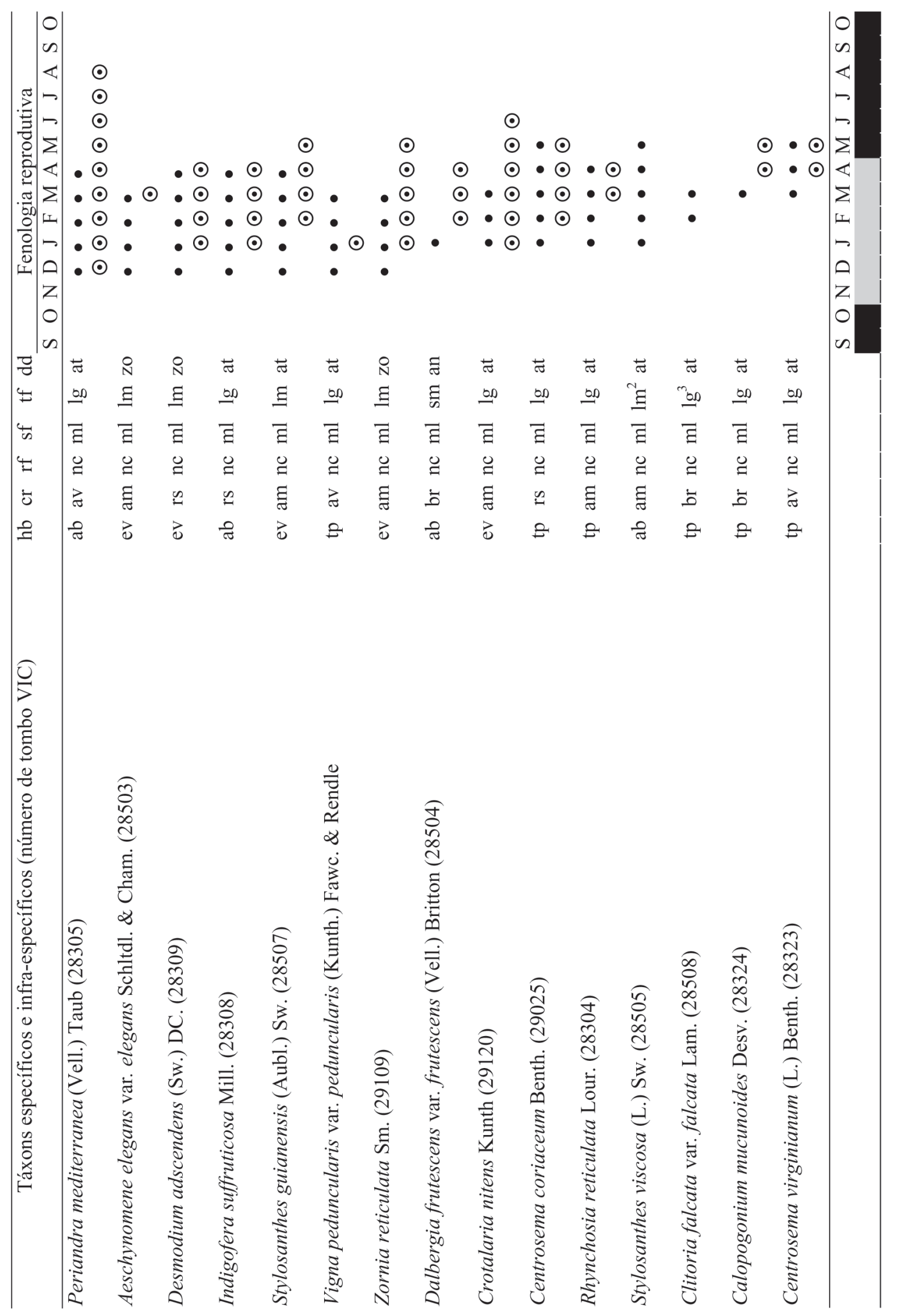




\begin{tabular}{|c|c|c|}
\hline Táxons específicos e infra-específicos (número de tombo VIC) & hb cr rf $\mathrm{sf}$ tf $\mathrm{dd}$ & Fenologia reprodutiva \\
\hline & & $\mathrm{S}$ O N D J F M A M J J A S O \\
\hline Crotalaria micans Link (28701) & $\mathrm{ab}$ am $\mathrm{nc} \mathrm{ml} \mathrm{lg}^{4}$ at & - \\
\hline Desmodium barbatum (L.) Benth. (28325) & ev av nc $\mathrm{ml} \operatorname{lm}$ zo & $\bullet$ \\
\hline Desmodium affine Schltdl. (28326) & ev rs nc $\mathrm{ml} \operatorname{lm} \mathrm{zo}$ & • $\odot$ \\
\hline Desmodium incanum DC. (28328) & ev rs nc $\mathrm{ml} \operatorname{lm} \mathrm{zo}$ & $\dot{\odot} \odot$ \\
\hline Desmodium uncinatum (Jacq.) DC. (28321) & ev rs nc $\mathrm{ml} \operatorname{lm} \mathrm{zo}$ & - $\bullet$ \\
\hline Swartzia oblata R.S. Cowan (28976) & $\mathrm{ab}$ br $\mathrm{nc} \mathrm{ml} \quad \mathrm{lc}^{5} \mathrm{zo}$ & $\bullet$ \\
\hline Machaerium aculeatum Raddi (28502) & $\ln 11^{6}-\mathrm{ml}^{6} \mathrm{sm}$ an & $\odot \odot \odot \odot$ \\
\hline Machaerium brasiliense Vogel (28307) & $a b \mathrm{~cm}^{6}-\mathrm{ml}^{6} \mathrm{sm}$ an & $\odot \odot \odot \odot \odot$ \\
\hline
\end{tabular}

1. Pennington (2003); 2. Brandão \& Costa (1979); 3. Fantz (1980); 4. Flores \& Miotto (2001); 5. Mansano \& Tozzi (2004); 6. Sartori \& Tozzi (1998). 


\section{Fenologia}

A floração das espécies de Leguminosae estudadas ocorreu durante todo o período de estudo (Fig. 1b) e os dois maiores picos dessa fenofase foram registrados na estação chuvosa, em janeiro e em março (44,4 e 66,6\% dos táxons, respectivamente). $\mathrm{O}$ pico em março foi devido, principalmente, à floração das espécies de Papilionoideae (Fig. 1b). A floração foi correlacionada com a precipitação $(r=0,67$; $\mathrm{p}<0,01)$ e com a temperatura $(\mathrm{r}=0,79 ; \mathrm{p}<$ $0,01)$.

O padrão fenológico de floração mais freqüente foi do tipo anual, observado em $88,9 \%$ dos táxons, sendo $35,5 \%$ de floração intermediária e $26,7 \%$ de estendida ou breve (Tab. 1). A floração subanual foi registrada em Mimosa aurivillus var. aurivillus; a floração contínua ocorreu em Chamaecrista dentata e C. mucronata. Machaerium brasiliense e M. aculeatum parecem apresentar floração supra-anual, uma vez que os indivíduos foram observados apenas em frutificação (Tab. 1).

Dentre os 45 táxons, Andira surinamensis, Clitoria falcata var. falcata, Crotalaria micans, Stylosanthes viscosa e Swartzia oblata não frutificaram no período de estudo (Tab. 1). A frutificação das demais espécies de Leguminosae estudadas ocorreu durante todo o período de estudo. O maior pico de frutificação foi observado em abril (Fig. 1c), período de transição entre a estação chuvosa e seca (Fig. 1a), quando $57,7 \%$ dos táxons apresentaram frutos, a maioria da subfamília Papilionoideae (58\%, Fig. 1c). A frutificação foi correlacionada com a temperatura $(\mathrm{r}=0,85$; $\mathrm{p}<0,01)$. A maioria dos táxons $(65,8 \%)$ apresentou frutificação anual intermediária. A frutificação anual breve foi representada por $13,2 \%$ dos táxons, a anual estendida por $18,4 \%$ e a contínua por $2,6 \%$ (Tab. 1).

Em cada subfamília, as espécies apresentaram a tendência de floração e frutificação anual: $70 \%$ e $90 \%$ das Caesalpinioideae, $89 \%$ e $100 \%$ das Papilionoideae e $62 \%$ e $100 \%$ das Mimosoideae, respectivamente. Em Caesalpinioideae, o pico de floração ocorreu em março para $80 \%$ das espécies (Fig. 1b) e o de frutificação, em março, julho e agosto, para 60\% (Tab. 1, Fig. 1c). Em Chamaecrista foi observada sobreposição da floração, além da floração sequencial entre Chamaecrista hedysaroides, C. rotundifolia var. rotundifolia e $C$. rotundata var. grandistipula (Tab. 1). Também houve sobreposição de floração entre as espécies de Senna e entre espécies de Chamaecrista e Senna (Tab. 1). Apenas Bauhinia ungulata var. cuiabensis apresentou exclusivamente flores na estação seca (Tab. 1).

Entre as espécies de Mimosoideae, o maior número de espécies em floração ocorreu em janeiro (55\%, Fig. 1b) e o de frutificação, em abril (67\%, Fig. 1c). Mimosa aurivillus var. aurivillus, M. ourobrancoënsis e Inga vulpina floresceram exclusivamente na estação seca (Tab. 1).

Em Papilionoideae, assim como verificado em Caesalpinioideae, a maior porcentagem de espécies em floração foi observada em março (73\%, Fig. 1b) e a de frutificação, assim como verificado em Mimosoideae, em abril (65\%, Fig. 1c). As espécies floresceram principalmente na estação chuvosa e Swartzia oblata foi a única que floresceu na estação seca (Tab. 1). Houve sobreposição de floração entre espécies congêneres e entre espécies de diferentes gêneros (Tab. 1). Calopogonium mucunoides, Centrosema virginianum e Desmodium uncinatum foram as espécies que apresentaram frutificação exclusivamente na estação seca (Tab. 1).

\section{Síndromes florais e de dispersão}

A maioria das espécies estudadas $(88,8 \%)$ apresentou características florais da síndrome de melitofilia, tais como corolas com cores amarelas, róseas ou violáceas (Tab. 1), zigomorfas, papilionadas ou abertas, e em Chamaecrista spp. e Senna spp. anteras poricidas. Dentre os táxons melitófilos, registraram-se flores nectaríferas e poliníferas (Tab. 1). As nectaríferas estão representadas, especialmente, pelas espécies de Papilionoideae 
(53\% das espécies estudadas) e as poliníferas pelas espécies de Caesalpinioideae (20\%, Tab. 1).

Nas espécies de Caesalpinioideae, as flores de Senna e Chamaecrista variam de zigomorfas a assimétricas, amarelas, oferecem como recurso o pólen e são polinizadas por abelhas grandes (Bombus morio coletada em C. dentata), estando a melitofilia representada em $90 \%$ dos táxons. A quiropterofilia foi registrada apenas em Bauhinia ungulata var. cuiabensis, que apresenta flores actinomorfas, noturnas, brancas e nectaríferas.

As flores das espécies de Mimosoideae são actinomorfas, reunidas em inflorescências globosas, tipo pincel. A corola é verde, gamopétala e tubulosa. O androceu é composto por 2-50 estames, com filetes longos, róseos, amarelos ou alvos. Em Mimosa as flores são melitófilas (66,6\% dos táxons), têm antese diurna e o recurso oferecido aos visitantes é o pólen (Tab. 1). Em Mimosa montis-carasae, além de diversas abelhas pequenas, também foi observada a presença de abelhas do gênero Bombus. Inga barbata e Abarema langsdorfii apresentaram flores com filetes brancos, antese noturna, e o recurso, é o néctar (Tab. 1). Essas características assemelham-se às de espécies polinizadas por esfingídeos, estando a esfingofilia representada por $22,4 \%$ dos táxons de Leguminosae. Inga vulpina, com flores vistosas, de antese diurna $\mathrm{e}$ nectaríferas, apresenta características de espécies psicófilas (11\%).

Nas espécies de Papilionoideae, as flores são fortemente zigomorfas, papilionadas, ou seja, possuem uma pétala superior, denominada vexilo, duas pétalas laterais, as alas, e duas inferiores, que juntas formam a carena, geralmente envolvendo o androceu e gineceu, exceto Swartzia oblata (possui corola aberta). As papilionadas apresentam-se ressupinadas em Centrosema, Periandra e Clitoria, e a corola possui colorações variadas (Tab. 1). A antese é diurna e oferecem néctar aos visitantes (Tab. 1). A maioria é melitófila (96\%, Tab. 1). Centrosema coriaceum e Periandra mediterranea foram visitadas por abelhas grandes (Bombus morio). A ornitofilia foi observada em Camptosema scarlatinum var. pohlianum, espécie com corola vermelha (Tab. 1).

As espécies autocóricas, que apresentam frutos do tipo legume, folículo ou craspédio (Tab. 1), representaram $66,6 \%$ do total dos táxons que frutificaram. As zoocóricas, que apresentam lomentos ou legumes nucóides (Tab. 1), representaram $26,7 \%$ dos táxons e as anemocóricas, que apresentam sâmaras ou legumes samaróides (Tab. 1), representaram 6,7\%. Entre as Caesalpinioideae, $100 \%$ dos táxons são autocóricos. Nas Mimosoideae, 66,6\% dos táxons são autocóricos e 33,4\%, zoocóricos e, em Papilionoideae a autocoria está representada por $54 \%$ dos táxons, seguida pela zoocoria $(34,5 \%)$ e pela anemocoria $(11,5 \%)$.

Entre junho e setembro/2004 na estação seca, a frutificação foi representada apenas por táxons autocóricos, principalmente pelas espécies de Caesalpinioideae, havendo correlação com a temperatura $(\mathrm{r}=0,71 ; \mathrm{p}<$ 0,01). A maior proporção de espécies zoocóricas ocorreu durante a estação chuvosa, entre janeiro e abril/2004. A zoocoria foi correlacionada com a temperatura $(r=0,94 ; p$ $<0,01)$ e com a precipitação $(\mathrm{r}=0,69 ; \mathrm{p}<$ $0,01)$. A anemocoria foi freqüente no final da estação seca, entre setembro e novembro/2003 (Fig. 2) e foi correlacionada com a precipitação $(\mathrm{r}=0,53 ; 0.01 \leq \mathrm{p}<0,05)$.

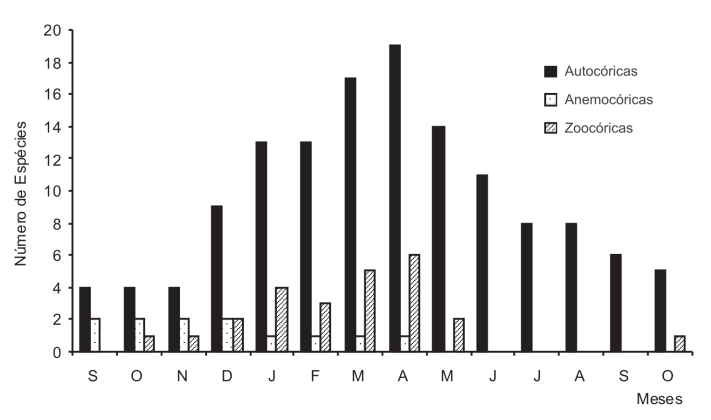

Figura 2 - Variações mensais na porcentagem de síndromes de dispersão dos táxons encontrados com frutos no período de setembro/2003 a outubro/2004.

Figure 2 - Monthly variation of the dispersion syndromes (percentage) presented by the frutifying species from september/2003 to october/2004. 
Tabela 2 - Altura média (cm) e desvio padrão dos estames e pistilos das espécies de Chamaecrista estudadas nos campos rupestres do Parque Estadual do Itacolomi, Minas Gerais, Brasil. ( $\mathrm{n}=$ número de flores medidas por espécie; $\mathrm{dp}=$ diferença proporcional entre as alturas do pistilo e estames).

Table 2 - Mean height $(\mathrm{cm})$ and standard deviations of stamens and pistils of Chamaecrista species surveyed in the "campos rupestres" of Parque Estadual do Itacolomi, Minas Gerais, Brasil. ( $\mathrm{n}=$ number of measured flowers per species; $\mathrm{dp}=$ proporcional difference between the height values of pistil and stamens).

\begin{tabular}{lcccc}
\hline Espécie & n & \multicolumn{2}{c}{ Altura e desvio padrão } \\
& & Estames & Pistilo & dp \\
\hline
\end{tabular}

Com deposição indireta de pólen

\begin{tabular}{lllll} 
Chamaecrista dentata & 24 & $0,66 \pm 0,06$ & $1,44 \pm 0,12$ & 2,18 \\
Chamaecrista hedysaroides & 5 & $0,58 \pm 0,05$ & $1,2 \pm 0,35$ & 2,06 \\
Chamaecrista mucronata & 7 & $0,81 \pm 0,06$ & $1,42 \pm 0,19$ & 1,75 \\
$\begin{array}{llll}\text { Chamaecrista rotundata var. grandistipula } \\
\text { Com deposição direta de pólen }\end{array}$ & 5 & $1,01 \pm 0,19$ & $2,3 \pm 0,20$ & 2,27 \\
Chamaecrista desvauxii var. langsdorfii & 10 & $0,86 \pm 0,09$ & $0,66 \pm 0,09$ & 0,76 \\
Chamaecrista rotundifolia var. rotundifolia & 9 & $0,36 \pm 0,06$ & $0,38 \pm 0,05$ & 1,05 \\
Chamaecrista trichopoda & 12 & $0,76 \pm 0,10$ & $0,99 \pm 0,16$ & 1,30 \\
\hline
\end{tabular}

\section{Padrões de deposição do pólen em Chamaecrista}

Os táxons de Chamaecrista foram divididos em dois grupos devido às diferenças entre as alturas dos estames e do pistilo (Tab. 2). No primeiro grupo, denominado padrão de deposição direta do pólen, representado por $C$. trichopoda, C. desvauxii var. langsdorfii e $C$. rotundifolia var. rotundifolia, a diferença proporcional entre as alturas do pistilo e dos estames foi menor ( $d p=0,76$ a 1,30, Tab. 2). Nessas espécies, o pólen é liberado das anteras diretamente no corpo do polinizador durante o processo de vibração da flor pelas abelhas. A pétala vexilar interna desses táxons é estendida (Fig. 3a-c), diferentemente do observado no segundo grupo.

No segundo grupo, denominado padrão de deposição indireta do pólen (di), representado por C. dentata, C. hedysaroides. C. mucronata e C. rotundata var. grandistipula, todas enantiostílicas, registrou-se maior diferença proporcional entre a altura do pistilo e dos estames $(\mathrm{dp}=1,75$ e 2,27, Tab. 2). Essas espécies apresentam a pétala vexilar interna modificada, em grau variável (Fig. 3d-g), que envolve as anteras e recebe o pólen ventralmente, durante a vibração das flores pelas abelhas. O pólen passa por toda a extensão ventral da pétala e é depositado, por via indireta, no corpo dos polinizadores. Desse modo, as maiores diferenças de altura registradas entre anteras e estigmas são superadas pela pétala vexilar, que funciona como um prolongamento dos estames. O pólen tende a ser depositado em locais específicos do corpo das abelhas; em flores de $C$. dentata, por exemplo, os grãos de pólen foram depositados na região proximal das asas de Bombus morio. De acordo com a espécie, a pétala vexilar interna apresenta-se cuculada (Fig. 3d-e) ou tubular (Fig. 3f-g), estando sua porção mais distal oposta ao estigma.

\section{Discussão}

A correlação da floração com a precipitação e temperatura corrobora o proposto por Rathcke \& Lacey (1985); esses autores afirmaram que, em áreas tropicais com clima sazonal, a maioria das ervas e arbustos floresce na estação chuvosa. Outros 

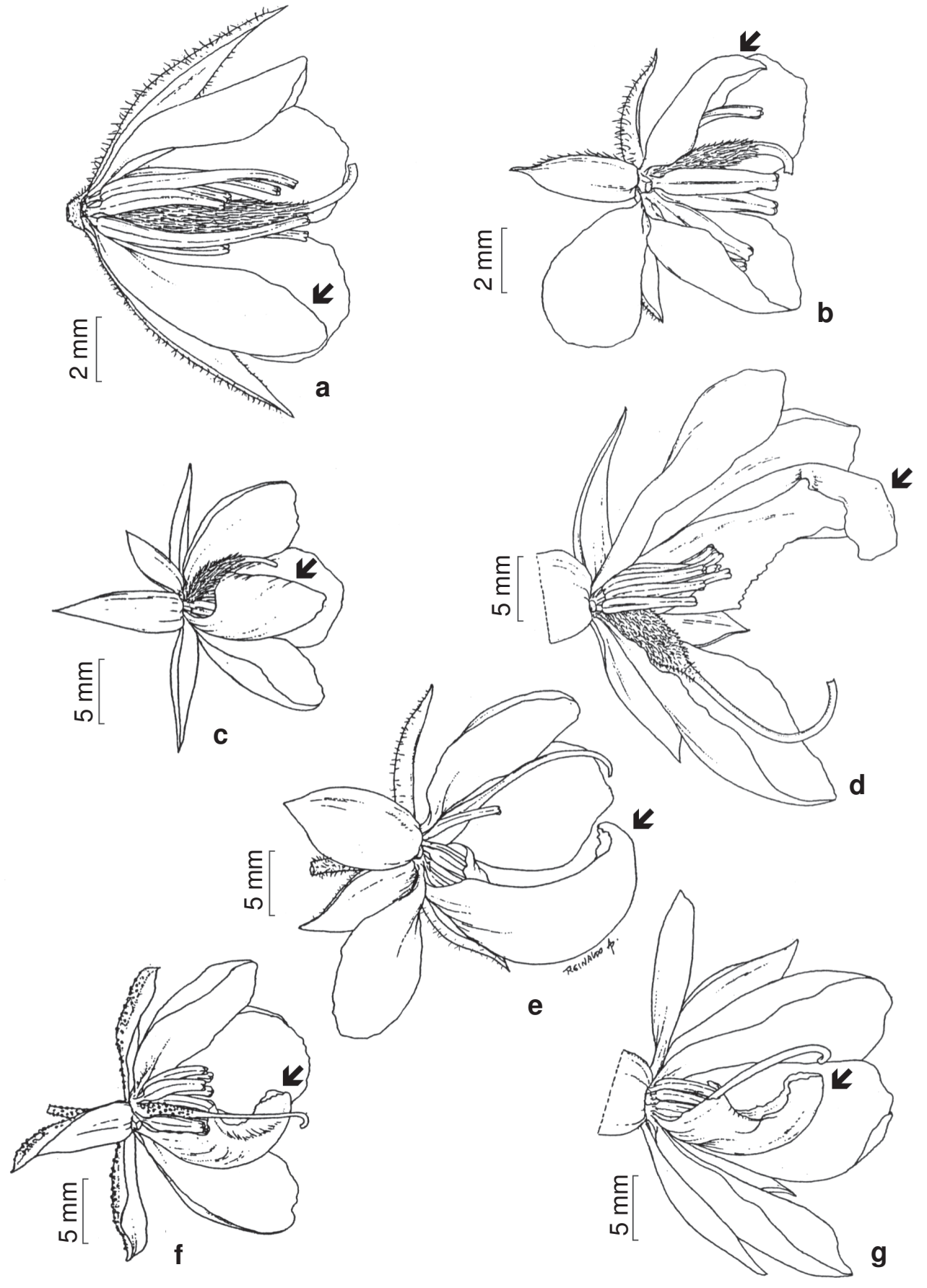

Figura 3 - Flores das espécies de Chamaecrista; a posição da pétala vexilar interna é indicada por seta - a. C. trichopoda; b. C. rotundifolia var. rotundifolia; c. C. desvauxii var. langsdorfii; d. C. rotundata var. grandistipula; e. C. mucronata; f. C. hedysaroides; g. C. dentata. Nas espécies C. rotundata var. grandistipula, C. mucronata, C. hedysaroides e $C$. dentata a pétala vexilar interna é modificada.

Figure 3 - Flowers of Chamaecrista species; the position of the internal vexillary petal is indicated by arrow - a. C. trichopoda; b. C. rotundifolia var. rotundifolia; c. C. desvauxii var. langsdorfii; d. C. rotundata var. grandistipula; e. C. mucronata; f. C. hedysaroides; g. C. dentata. On the species $C$. rotundata var. grandistipula, C. mucronata, C. hedysaroides and C. dentata the internal vexillary petal is modified. 
trabalhos têm obtido resultados semelhantes em espécies de cerrado, mata, campo de altitude, além dos campos rupestres (Barros \& Caldas 1980; Mantovani \& Martins 1988; Pedroni et al. 2002; Carvalho \& Oliveira 2003; Mantovani et al. 2003; Dutra et al. 2005; Munhoz \& Felfili 2005; Freitas \& Sazima 2006; Conceição et al. 2007).

O predomínio de espécies com floração anual também ocorreu em espécies de Papilionoideae de campo ferruginoso (Dutra et al. 2005) e em Leguminosas arbóreas de mata (Mendonça-Filho 1995) e de cerrado (Goulart et al. 2005), reforçando a tendência desse padrão de floração ser o mais comum entre as espécies tropicais (Newstrom et al. 1994).

Embora não tenha havido correlação significativa entre frutificação e precipitação, diferentemente do observado por Dutra et al. (2005) nos campos ferruginosos do PEI, o pico de frutificação ocorreu no período de transição da estação chuvosa para a seca (abril). Isso pode estar relacionado à presença predominante de espécies autocóricas, que se correlacionam com a temperatura, e necessitam da desidratação do pericarpo para a liberação das sementes. Além disso, a maturação e dispersão dos frutos na estação seca aumentam a probabilidade de germinação de sementes no próximo período chuvoso (Mantovani \& Martins 1988; Felfili et al. 1999). A frutificação, principalmente anual intermediária, assemelha-se ao verificado em Copaifera langsdorffii Desf., em floresta semidecídua (Pedroni et al. 2002) e em espécies de Papilionoideae, de campo ferruginoso (Dutra et al. 2005).

As proporções de espécies autocóricas, anemocóricas e zoocóricas encontradas foram similares ao observado em afloramentos rochosos da Chapada Diamantina (Conceição et al. 2007). A dispersão de espécies autocóricas e anemocóricas na estação seca e das zoocóricas na estação chuvosa também foi relatada por diversos autores em áreas de mata e cerrado (Mantovani \& Martins 1988; Morellato et al. 1989; Batalha \& Mantovani
2000). Esses autores relacionaram o período de dispersão dos diásporos com as variações climáticas - na estação seca, são mais comuns os frutos anemocóricos e autocóricos, devido aos fortes ventos e o ar mais seco, que favorecem a dispersão. Na estação chuvosa, os frutos zoocóricos apresentam mais chances de dispersão, pois a alta umidade aumenta a duração, a atratividade e a diversidade de dispersores (Batalha \& Mantovani 2000).

A melitofilia como síndrome floral dominante em espécies de Leguminosas dos campos rupestres do PEI assemelha-se ao verificado em espécies da caatinga, de matas, de dunas tropicais, de campos de altitude, de afloramentos rochosos e dos cerrados (Gottsberger et al. 1988; SilberbauerGottsberger \& Gottsberger 1988; Costa \& Ramalho 2001; Machado \& Lopes 2004; Freitas \& Sazima 2006; Conceição et al. 2007; Yamamoto et al. 2007), inclusive na savana venezuelana (Ramírez 2004). A alta porcentagem de espécies melitófilas deve-se às flores papilionadas (Papilionoideae) e às com anteras poricidas, tais como Cassia, Senna e Chamaecrista (SilberbauerGottsberger \& Gottsberger 1988). Além disso, as altitudes elevadas e a ocorrência de ventos fortes dos campos rupestres (Giulietti et al. 1987), restringem a atividade de muitos animais antófilos (Gottsberger et al. 1988; Faria 1994).

Clitoria falcata var. falcata, Periandra mediterranea, Centrosema spp., Chamaecrista spp. e Senna spp. parecem ser importantes fontes alimentares para abelhas grandes, pois fornecem, em conjunto, recursos florais ao longo do ano, auxiliando na manutenção desses insetos no PEI. Indivíduos de Bombus spp., por exemplo, encontram-se em atividade durante todo o ano e visitam flores de espécies de diferentes famílias, inclusive de Leguminosae, em busca de pólen e, ou néctar (Cortopassi-Laurino et al. 2003).

A sincronia de floração entre Chamaecrista dentata, C. mucronata e Senna reniformis e a floração sequencial entre as espécies de Chamaecrista, além de $S$. 
pendula, associada às semelhanças morfológicas de suas flores e de seu hábitat, parecem promover, entre esses táxons, uma "série de reposição" (sensu Macior 1971), ou seja, espécies distintas resultam, em conjunto, numa "única" floração, por um longo período. Assim, há formação e retenção de uma "imagem de procura" alimentar por parte de polinizadores em comum (Thomson 1980), e ao mesmo tempo, disponibilidade, durante todo o ano, de recurso floral (pólen) aos seus polinizadores. As Caesalpinioideae, principalmente espécies de Senna e Chamaecrista, também representam importante fonte de pólen na caatinga (Machado \& Lopes 2004).

A esfingofilia em Abarema langsdorfii e Inga barbata reforçam a polinização de muitos gêneros da tribo Ingeae, inclusive Inga, por mariposas (esfingídeos), como observado por Koptur (1983) e Neto et al. (2007). Esta síndrome, embora comumente citada para espécies de Leguminosae-Mimosoideae, é ainda pouco estudada (Arroyo 1981; Bawa 1990). A psicofilia é comum em flores nectaríferas que exibem partes florais com cores brilhantes (Bawa 1990), como as de Inga vulpina.

A baixa representatividade de espécies quiropterófilas, apenas registrada em Bauhinia ungulata var. cuiabensis, corrobora os estudos de Conceição et al. (2007), realizado na Chapada Diamantina. A quiropterofilia é encontrada em muitas famílias e é bem conhecida no gênero Bauhinia (Bawa 1990). As espécies quiropterófilas geralmente florescem no período de seca e o néctar dessas plantas parece ser essencial para os morcegos, pois nesse período há escassez de outros alimentos (Silva \& Peracchi 1999). A ornitofilia, registrada apenas em Camptosema scarlatinum var. pohlianum, tem sido observada em diferentes grupos de Leguminosae e em Papilionoideae, ocorre principalmente nas tribos Sophoreae e Phaseoleae, na qual encontra-se o gênero Camptosema (Arroyo 1981).

A divisão das espécies de Chamaecrista em dois grupos, considerando as diferenças proporcionais entre alturas de anteras e estigma, possibilitou a visualização de duas estratégias de polinização, envolvendo: 1) a deposição direta do pólen, da antera ao corpo do polinizador, e 2) a deposição indireta do pólen, na qual a pétala vexilar interna é modificada e funciona como um prolongamento dos estames, sendo responsável pela deposição do pólen no corpo do polinizador. A função da pétala modificada como uma extensão dos estames é óbvia (Gottsberger \& SilberbauerGottsberger 1988) e os resultados do presente estudo ampliam registro de espécies desse gênero com esse mecanismo de polinização. Nessas plantas, as abelhas grandes, principalmente Bombus spp., parecem ser os polinizadores efetivos (Gottsberger \& Silberbauer-Gottsberger 1988; presente estudo). A pétala vexilar modificada é uma característica floral adaptativa que favorece a polinização (Gottsberger et al. 1988), pois funciona como um extensor do conjunto de estames da flor, resultando na deposição do pólen em áreas específicas do corpo dos polinizadores. Além disso, essa característica e a enantiostilia reduzem as chances de autopolinização.

Analisando-se os resultados como um todo, verifica-se que os padrões fenológicos de floração e frutificação das espécies de Leguminosae dos campos rupestres do Parque Estadual do Itacolomi, em geral, seguem os padrões já descritos na literatura, principalmente para áreas de cerrado e outras vegetações de altitude, e mostram-se sazonais, com a floração ocorrendo principalmente na estação chuvosa e a frutificação no final da estação chuvosa e início da seca. A dispersão dos diásporos, a maioria autocóricos, ocorre especialmente na estação seca. Esses resultados indicam que a sazonalidade climática é importante para a comunidade estudada.

A melitofilia é a síndrome mais freqüente, assim como em outras áreas com clima sazonal, e reforça a importância da família Leguminosae como uma das maiores fontes de recursos alimentares (néctar e pólen) para as abelhas. 


\section{Agradecimentos}

Os autores agradecem ao Instituto Estadual de Florestas (IEF), pela autorização concedida para a realização das coletas; aos funcionários do Parque Estadual do Itacolomi, pelo auxílio nos trabalhos de campo; à Profa. Maria Cristina T. B. Messias (UFOP), pelo auxílio com as análises estatísticas; ao Dr. Luciano Paganucci de Queiroz (UEFS), pelas críticas e sugestões ao manuscrito; ao Dr. Lúcio Antônio de Oliveira Campos, pela identificação das abelhas; e ao Reinaldo A. Pinto, pela elaboração das ilustrações.

\section{REFERÊNCIAS BibLIOGRÁFICAS}

Arroyo, M. T. K. 1981. Breeding systems and pollination biology in Leguminosae. In: Polhill, R. M. \& Raven, P. H. (eds.). Advances in legume systematics. Part II. Royal Botanic Gardens, Kew. Pp.723-769.

Barros, M. A. G. \& Caldas, L. S. 1980. Acompanhamento de eventos fenológicos apresentados por cinco gêneros nativos do Cerrado (BrasíliaDF). Brasil Florestal 42: 7-14.

Batalha, M. A.; Aragaki, S. \& Mantovani, W. 1997. Variações fenológicas das espécies do cerrado em Emas (Pirassununga, SP). Acta Botanica Brasilica 11(1): 61-78.

Batalha, M. A. \& Mantovani, W. 2000. Reproductive phenological patterns of Cerrrado plant species at the Pé-deGigante Reserve (Santa Rita do Passa Quatro, SP, Brazil): a comparison between the herbaceous and woody floras. Revista Brasileira de Biologia 60(1): 129-145.

Bawa, K. S. 1990. Plant-pollinator interactions in tropical rain forests. Annual Review of Ecology and Systematics 21: 399-422.

Brandão, M. \& Costa, N. M. S. 1979. O gênero Stylosanthes Sw. no Brasil. EPAMIG, Belo Horizonte, 108p.

Carmo, R. M. \& Franceschinelli, E. V. 2002. Polinização e biologia floral de Clusia arrudae Planchon \& Triana (Clusiaceae) na Serra da Calçada, município de Brumadinho, MG. Revista Brasileira de Botânica 25(3): 351-360.
Carvalho, D. A. \& Oliveira, P. E. 2003. Biologia reprodutiva e polinização de Senna sylvestris (Vell.) H.S. Irwin \& Barneby (Leguminosae, Caesalpinioideae). Revista Brasileira de Botânica 26(3): 319-328.

Conceição, A. A.; Funch, L. S. \& Pirani, J. R. 2007. Reproductive phenology, pollination and seed dispersal syndromes on sandstone outcrop vegetation in the "Chapada Diamantina", northeastern Brazil: population and community analyses. Revista Brasileira de Botânica 30(3): 475-485.

Cortopassi-Laurino, M.; Knoll, F. R. N. \& Imperatriz-Fonseca, V. L. 2003. Nicho trófico e abundância de Bombus morio e Bombus atratus em diferentes biomas brasileiros. In: Melo, G. A. R. \& Santos, I. A. (eds.). Apoidea neotropica: homenagem aos 90 anos de Jesus Santiago Moure. Ed. UNESC, Criciúma. Pp. 285-295.

Costa, J. A. S. \& Ramalho, M. 2001. Ecologia da polinização em ambiente de duna tropical (APA do Abaeté, Salvador, Bahia, Brasil). Sitientibus, série Ciências Biológicas 1(2): 141-153.

Drummond, G. M.; Martins, C. S.; Machado, A. B. M.; Sebaio, F. A. \& Antonini, Y. 2005. Biodiversidade em Minas Gerais: um atlas para sua conservação. Fundação Biodiversitas, Belo Horizonte, 222p.

Dutra, V. F. 2005. Leguminosae Adans. nos campos rupestres do Parque Estadual do Itacolomi, Minas Gerais, Brasil: florística, preferência por habitat, aspectos reprodutivos e distribuição geográfica. Dissertação de Mestrado, Universidade Federal de Viçosa, Viçosa, 171p.

Dutra, V. F; Garcia, F. C. P.; Lima, H. C. \& Queiroz, L. P. 2008. Diversidade florística de Leguminosae Adans. em áreas de campos rupestres. Megadiversidade 4(1-2): 145-153.

Dutra, V. F.; Messias, M. C. T. B. \& Garcia, F. C. P. 2005. Papilionoideae (LeguminosaePapilionoideae) dos campos ferruginosos do Parque Estadual do Itacolomi, MG, Brasil: florística e fenologia. Revista Brasileira de Botânica 28(3): 493-504. 
Endress, P. K. 1994. Diversity and evolutionary biology of tropical flowers. Cambridge University Press, Cambridge, 511p.

Faegri, K. \& van der Pijl, L. 1979. The principles of pollination ecology. Pergamon Press, Oxford, 242p.

Fantz, P. R. 1980. Flora of Panama. Annals of the Missouri Botanical Garden 67: 582-593.

Faria, G. M. 1994. A flora e a fauna apícola de um ecossistema de campo rupestre, Serra do Cipó - MG, Brasil: composição, fenologia e suas interações. Tese de Doutorado, Universidade Estadual Paulista, Rio Claro, 239p.

Felfili, J. M.; Silva-Junior, M. C.; Dias, B. J. \& Rezende, A. V. 1999. Estudo fenológico de Stryphnodendron adstringens (Mart.) Coville no cerrado sensu stricto da Fazenda Água Limpa no Distrito Federal, Brasil. Revista Brasileira de Botânica 22(1): 83-90.

Flores, A. S. \& Miotto, S. T. S. 2001. Gênero Crotalaria L. (Leguminosae-Faboideae) na Região Sul do Brasil. Iheringia, série Botânica 55: 189-247.

Fournier, L. A. 1976. Observaciones fenologicas en el bosque húmedo de premontano de San Pedro de Montes de Oca, Costa Rica. Turrialba 26(1): 54-59.

Frankie, G. W.; Baker, H. G. \& Opler, P. A. 1974. Tropical plant phenology: applications for studies in community ecology. In: Lieth, H. (ed.). Phenology and seasonality modeling. Springer Verlag, Berlim. Pp. 287-296.

Freitas, L. \& Sazima, M. 2006. Pollination biology in a tropical high-altitude grassland in Brazil: interactions at the community level. Annals of the Missouri Botanical Garden 93: 465-516.

Giulietti, A. M.; Menezes, N. L.; Pirani, J. R.; Meguro, M. \& Wanderley, M. G. L. 1987. Flora da Serra do Cipó: caracterização e lista das espécies. Boletim de Botânica da Universidade de São Paulo 9: 1-151.

Gottsberger, G.; Camargo, J. M. F. \& Silberbauer-Gottsberger, I. 1988. A bee- pollinated tropical community: The beach dune vegetation of Ilha de São Luís, Maranhão, Brasil. Botanische Jahrbücher für Systematik, Pflanzengeschichte und Pflanzengeographie 109: 469-500.

Gottsberger, G. \& Silberbauer-Gottsberger, I. 1988. Evolution of flower structures and pollination in Neotropical Cassiinae (Caesalpiniaceae) species. Phyton (Austria) 28(2): 293-320.

Goulart, M. F.; Filho, J. P. L. \& Lovato, M. B. 2005. Phenological variation within and among populations of Plathymenia reticulata in Brazilian Cerrado, the Atlantic Forest and transitional sites. Annals of Botany 96: 445-455.

Harley, R. M. 1995. Introduction. In: Stannard, B. L. (ed.). Flora of the Pico das Almas, Chapada Diamantina, Bahia, Brazil. Royal Botanical Gardens, Kew. Pp. 1-40.

Koptur, S. 1983. Flowering phenology and floral biology of Inga (Fabaceae: Mimosoideae). Systematic Botany 8(4): 354-368.

Machado, I. C. \& Lopes, A. V. 2004. Floral traits and pollination systems in the Caatinga, a Brazilian Tropical Dry Forest. Annals of Botany 94(3): 365-376.

Macior, L.W. 1971. Coevolution of plants and animals-systematic insights from plantinsect interactions. Taxon 20: 17-28.

Madeira, J. A. \& Fernandes, G. W. 1999. Reproductive phenology of sympatric taxa of Chamaecrista (Leguminosae) in Serra do Cipó, Brazil. Journal of Tropical Ecology 15: 463-479.

Mantovani, M.; Ruschel, A. R.; Reis, M. S.; Puchalski, A. \& Nodari, R. O. 2003. Fenologia reprodutiva de espécies arbóreas em uma formação secundária da Floresta Atlântica. Revista Árvore 27(4): 451-458.

Mantovani, W. \& Martins, F. R. 1988. Variações fenológicas das espécies do cerrado da Reserva Biológica de Moji Guaçu, estado de São Paulo. Revista Brasileira de Botânica 11: 101-112. 
Mansano, V. F. \& Tozzi, A. M. G. A. 2004. Swartzia (Leguminosae, Papilionoideae, Swartzieae s.1.) na Reserva Natural da Companhia Vale do Rio Doce, Linhares, ES, Brasil. Rodriguésia 55(85): 95-113.

Mendonça-Filho, C. V. 1995. Fenologia de Leguminosas arbóreas da Estação Biológica de Caratinga, Caratinga - MG. Dissertação de Mestrado. Universidade Federal de Minas Gerais, Belo Horizonte, 100p.

Menezes, N. L. \& Giulietti, A. M. 1986. Campos rupestres - paraíso botânico na Serra do Cipó. Ciência Hoje 5(25): 38-44.

Messias, M. C. T. B.; Dias, S. J.; Roschel, M. B.; Sousa, H. C. \& Matos, A. M. 1997. Levantamento florístico das matas e distribuição de algumas espécies endêmicas da região na área do Parque do Itacolomi. Relatório técnico. UFOP/ BIRD/IEF-Profloresta, Ouro Preto, 151p.

Morellato, L. P. C.; Rodrigues, R. R.; LeitãoFilho, H. F. \& Joly, C. A. 1989. Estudo comparativo da fenologia de espécies arbóreas de floresta de altitude e floresta mesófila semidecídua na Serra do Japi, Jundiaí, São Paulo. Revista Brasileira de Botânica 12: 85-98.

Morettin, P. A. \& Bussab, W. O. 2004. Estatística básica. 5ed. Saraiva, São Paulo, 526p.

Munhoz, C. B. R. \& Felfili, J. M. 2005. Fenologia do estrato herbáceosubarbustivo de uma comunidade de campo sujo na Fazenda Água Limpa no Distrito Federal, Brasil. Acta Botânica Brasilica 19(4): 979-988.

Neto, O. C.; Lopes, A. V. F. \& Machado, I. C. 2007. Ecologia da polinização de Inga striata (Benth.) (LeguminosaeMimosoideae) em um remanescente de mata Atlântica no Nordeste do Brasil. Revista Brasileira de Biociências 5(supl.1): 570-572.

Newstrom, L. E.; Frankie, G. W. \& Baker, H. G. 1994. A new classification for plant phenology based on flowering patterns in Lowland Tropical Rain Forest Trees at La Selva, Costa Rica. Biotropica 26(2): 141-159.

Pedroni, F.; Sanchez, M. \& Santos, F. A. M. 2002. Fenologia da copaíba (Copaifera langsdorffii Desf. Leguminosae, Caesalpinioideae) em uma floresta semidecídua no sudeste do Brasil. Revista Brasileira de Botânica 25(2): 183-194.

Pennington, R. T. 2003. Monograph of Andira (Leguminosae-Papilionoideae). Systematic Botany Monographs 64:1-143.

Peron, M. V. 1989. Listagem preliminar da flora fanerogâmica dos campos rupestres do Parque Estadual do Itacolomi - Ouro Preto/Mariana, MG. Rodriguésia 67(41): 63-69.

Ramírez, N. 2004. Ecology of pollination in a tropical Venezuelan savanna. Plant Ecology 173: 171-189.

Rathcke, B. \& Lacey E. P. 1985. Phenological patterns of terrestrial plants. Annual Review of Ecology and Systematics 16: 179-214.

Romero, R. 2002. Diversidade da flora dos Campos Rupestres de Goiás, Sudoeste e Sul de Minas Gerais. In: Araújo, E. L.; Moura, A. N.; Sampaio, E. V. S. B.; Gestinari L. M. S. \& Carneiro, J. M. T. (eds.). Biodiversidade, conservação e uso sustentável da flora do Brasil. Editora Universidade Federal de Pernambuco, Recife. Pp. 81-86.

Sartori, A. L. B. \& Tozzi, A. M. G. A. 1998. As espécies de Machaerium Pers. (Leguminosae - Papilionoideae Dalbergieae) ocorrentes no estado de São Paulo. Revista Brasileira de Botânica 21(3): 211-246.

Silberbauer-Gottsberger, I. \& Gottsberger, G. 1988. A polinização de plantas do cerrado. Revista Brasileira de Biologia 48(4): 651-663.

Silva, S. S. P. \& Peracchi, A. L. 1999. Visits of bats to flowers of Lafoensia glyptocarpa 
Koehne (Lythraceae). Revista Brasileira de Biologia 59(1): 19-22.

Stirton, C. H. 1981. Petal sculpturing in papilionoid legumes. In: Polhill, R. M. \& Raven, P. H. (eds.). Advances in legume systematics Royal Botanical Gardens, Kew. Pp. 771-788.

Thomson, J. D. 1980. Skewed flowering distributions and pollinator attraction. Ecology 61: 572-579.

van der Pijl, L. 1982. Principles of dispersal in higher plants. Springer-Verlag, New York, 215p.
Yamamoto, L. F.; Kinoshita, L. S. \& Martins, F. R. 2007. Síndromes de polinização e de dispersão em fragmentos da Floresta Estacional Semidecídua Montana, SP, Brasil. Acta Botanica Brasilica 21(3): 553-573.

Wunderlee Jr., J. M. 1997. The role of animal seed dispersal in accelerating native forest regeneration on degraded tropical lands. Forest Ecology and Management 99: 223-235. 